(2) Open Access Full Text Article

\title{
Assessment of the tolerability profile of an ophthalmic solution of $5 \%$ glycyrrhizin and copolymer PEG/PPG on healthy volunteers and evaluation of its efficacy in the treatment of moderate to severe blepharitis
}

This article was published in the following Dove Press journal:

Clinical Ophthalmology

8 July 2013

Number of times this article has been viewed

\section{Rita Mencucci \\ Eleonora Favuzza \\ Ugo Menchini}

Department of Surgery and Translational Medicine - Eye Clinic, University of Florence, Florence, Italy
Correspondence: Rita Mencucci Department of Surgery and Translational Medicine - Eye Clinic, University of Florence, Largo Brambilla 3, 50I 34 Florence, Italy

Email rita.mencucci@unifi.it
Purpose: To evaluate the tolerability on healthy volunteers and the efficacy on subjects affected by chronic moderate/severe blepharitis of a 5\% glycyrrhizin and copolymer poly(ethylene glycol)/ poly(propylene glycol)(PEG/PPG) ophthalmic solution.

Methods: The study was a randomized, controlled, open label, intra-patient monocentric study. It consisted of two different phases, the assessment of tolerability phase on 20 healthy volunteers, and the evaluation of the efficacy on 21 subjects affected by chronic moderate/severe blepharitis; the treatment period was 2 weeks, followed by 1-week of follow-up. In the efficacy phase, in both eyes, eyelid hygiene was also performed. At day $0,3,7,14$, and 21 a complete ophthalmological examination was performed. In the tolerability phase, signs of clinical toxicity were recorded and subject-reported symptoms were collected using a questionnaire. In the efficacy phase, global signs and symptoms of blepharitis scores were collected using standardized photographic scales and questionnaire. The statistical analysis was performed using the Wilcoxon signed-rank test.

Results: No ocular signs of drug toxicity were reported. During the treatment period for tolerability phase, there were statistically significant higher scores of tearing and ocular discomfort in the tolerability study group versus the tolerability control group. In the efficacy phase, differences between global scores of the two groups were statistically significant at day 0 (score of the efficacy study group was higher than the efficacy control group; $P=0.005)$ and at day 21 (score of the efficacy study group was lower than the efficacy control group $(P \leq 0.001)$. The difference of global scores at day $3,7,14$, and 21 versus day 0 was statistically significant in both groups. No serious adverse events occurred.

Conclusion: The 5\% glycyrrhizin ophthalmic solution was well tolerated in healthy volunteers and in patients with chronic moderate/severe blepharitis, and in association with eyelid hygiene showed good clinical anti-inflammatory activity that lasted after instillation suspension.

Keywords: glycyrrhizin, blepharitis, HMGB1

\section{Introduction}

Blepharitis is one of the most common ocular anterior segment disorders. ${ }^{1,2}$ Although it is an ocular inflammatory pathology that primarily involves the eyelid margin, it is also the most frequent cause of inflammation of other components of the ocular surface, such as the conjunctiva and the cornea. The presenting clinical features can be variable, depending on the association with coexisting ocular surface pathologies 
(eg, allergy or dry eye syndrome) and dermatologic conditions (eg, rosacea or seborrheic dermatitis). Blepharitis is usually a chronic condition, with recurrent acute exacerbations and relapses. ${ }^{1,2}$

Based on the anatomic classification of this condition, the clinical presentations can be divided in anterior blepharitis, involving the anterior lid margin, the base of the eyelashes, and the eyelash follicles, and posterior blepharitis, affecting the meibomian glands and gland orifices (meibomian gland dysfunction). ${ }^{1,3}$ Anterior blepharitis has been traditionally sub-classified on the basis of the presumed etiology in staphylococcal, seborrheic, and mixed blepharitis. ${ }^{1}$

Staphylococcal blepharitis is associated with Grampositive bacterial colonization of the eyelid margin, most frequently Staphylococcus aureus, that can cause ocular surface chronic inflammation by toxic and immune-mediated mechanisms; seborrheic blepharitis is due to the overproduction of sebum and is often associated with seborrheic dermatitis of the eyebrows or of the scalp. On the other hand, posterior blepharitis has a prominent inflammatory etiology and is characterized by plugging of the meibomian gland orifices and by altered gland secretions.

Despite this classification, the etiology is often multifactorial and the most common presentations may have features of both anterior and posterior involvement, making blepharitis one the most difficult anterior segment conditions to manage. ${ }^{1}$

Presently in the US there is no Food and Drug Administration (FDA)-approved therapy of blepharitis, and there is no sufficient evidence to strongly recommend any one a treatment option. ${ }^{1}$

Given the frequent presence of both bacterial and inflammatory etiology, the management of blepharitis consists principally in accurate eyelid hygiene, warm compresses, topical antibiotic, systemic antibiotics (in patients with meibomian gland dysfunction and rosacea), artificial lubricants, and topical corticosteroid. Topical corticosteroids should be used only in short courses at the minimal required dose to relieve signs and symptoms of acute exacerbations, due to their wellknown side-effects related to prolonged therapy. ${ }^{1}$

Recently, Cavone et $\mathrm{al}^{4}$ demonstrated the presence of an inflammatory mediator protein, high mobility group cox-1 (HMGB1) in tears of subjects affected by blepharitis in a 10 -fold higher level than in healthy control subjects.

HMGB1 is a protein of the alarmin family that is released in the extracellular space to activate the innate immune response, ${ }^{5,6}$ participates in tissue damage, and has demonstrated an active role in liver, lung, and other organ disorders, cancer, ${ }^{7-9}$ ocular physiology, and inflammatory/degenerative eye pathologies. ${ }^{10-12}$

It was also shown that the 18-beta-glycyrrhetic acid, the triterpene structure of the HMGB1-binding compound glycyrrhizin (extracted from the roots of the licorice plant Glycyrrhiza glabra), was capable of binding to HMGB1 and to alter the protein's pro-inflammatory properties, inhibiting HMGB1-dependent COX2 induction. ${ }^{13-14}$

Recently, a new 5\% glycyrrhizin and copolymer poly(ethylene glycol)/poly(propylene glycol) (PEG/PPG) ophthalmic solution (Saflogin ${ }^{\circledR}$ DMG Italia SRL, Rome, Italy) was made available in Italy.

Copolymer PEG/PPG is a thermosensitive copolymer that forms a gel when it contacts the eye surface, providing lubricating effects and a long-term permanence of the glycyrrhizin on the surface.

Given the significant presence of HGMB1 in the tears of subjects affected by blepharitis, and the potential role of its inhibitor glycyrrhizin in the management of external ocular inflammation, ${ }^{4}$ we evaluated the efficacy of the new medical device 5\% glycyrrhizin and copolymer PEG/PPG ophthalmic solution (DMG Italia SRL) on subjects affected by moderate/ severe blepharitis. We also assessed the tolerability profile of this solution in healthy volunteers.

\section{Material and methods Study design}

The study was a randomized, controlled, open label, intra-patient, monocentric study, involving 82 eyes of 41 patients. It was conducted between September 2011 and July 2012 in the Eye Clinic of Florence, Careggi Hospital, Department of Surgery and Translational Medicine, University of Florence, Italy.

This study, which adhered to the tenets of the Declaration of Helsinki, was conducted in accordance with the current International Conference on Harmonization-Good Clinical Practice (ICH-GCP) guidelines and was approved by the local ethics committee the 12th September 2011 (Prot 33693). The local ethics committee-approved written consent for the procedure and for the utilization of clinical data for scientific purposes were obtained from all subjects.

The study was made up of two different phases, the evaluation of tolerability profile of the ophthalmic solution on healthy volunteers, and the assessment of the efficacy on patients affected by moderate/severe chronic blepharitis.

\section{Tolerability phase}

The tolerability profile assessment consisted of a 2 week treatment period, followed by 1 -week of follow-up. 
Visits were performed at days 0 (Baseline), 3, 7, 14, and the follow-up visit at day 21 .

\section{Study population}

Twenty healthy adult volunteers of either sex were enrolled in the study ( $n=20 ; 40$ eyes) after signing the informed consent. Exclusion criteria were any sign or symptom of acute or chronic eye internal or external inflammation/infection at the inclusion visit or in the 2 previous weeks, history of herpetic keratitis (primary infection or recurrence), glaucoma and/or intra ocular pressure (IOP) $>22 \mathrm{mmHg}$, contact lens wearing in the 2 weeks prior to the inclusion visit or anticipated use during the study, any disease limiting eyelid closure function such as eye lid deformities, exophthalmia or facial palsy, dry eye syndrome, known endothelial corneal dystrophy, any ophthalmological treatment in the 2 weeks prior the inclusion visit or anticipated during the study, female volunteers who were pregnant, breast-feeding, or planning a pregnancy during the study, patients who received any other investigational agent within 30 days prior to study entry, patients not suitable for adequate follow up.

\section{Tolerability endpoints/safety measures}

Primary tolerability outcome parameters were the incidence of the following signs of drug toxicity: punctuate corneal staining, bulbar conjunctival hyperemia, or corneal infiltrates during the medication and follow-up phases, and the incidence of an IOP rose more than $4 \mathrm{mmHg}$.

Secondary endpoints were the incidence of ocular symptoms (itching, ocular discomfort, photophobia, tearing, or blurred vision). The time-course of the severity of the symptoms were assessed and compared to the baseline. The incidence of medication discontinuations due to clinically relevant drug toxicity was also recorded.

Any adverse events were collected and recorded at each visit.

\section{Inclusion visit (day 0)}

At the baseline visit, after signing informed consent, one eye from each participant $(n=20)$ was included in the tolerability study group, and the other eye $(n=20)$ in the tolerability control group. All eyes received treatment according to a computer-generated randomization schedule.

Medical and ocular history, concomitant medications and demographic data were recorded; women of childbearing potential underwent a urine pregnancy test. A complete ophthalmological evaluation was performed, including best corrected visual acuity (BCVA; LogMAR) measurement, slitlamp biomicroscopy examination with and without fluorescein staining, Goldmann tonometry, and dilated fundoscopic examination. The incidence of bulbar conjunctival hyperemia, corneal infiltrates, punctuate corneal staining was reported and graded on a four-point scale $(0=$ none, $1=$ mild, $2=$ moderate, $3=$ severe). Inclusion/exclusion criteria were reviewed, and a self-administering symptoms questionnaire was administered to volunteers. ${ }^{15}$ Subjects were asked to grade the intensity and the frequency of the following ocular symptoms, scored on a four-point scale $(0=$ none, $1=$ mild, 2 = moderate, 3 = severe): itching, ocular discomfort, photophobia, tearing, and blurred vision.

The study medication was dispensed and patients were instructed to instill two drops of ophthalmic solution three times a day in the study eye, starting the morning after the visit, while in the control eye no medication had to be administered.

\section{Visits after day 3, 7, I4 and 2 I}

Medical and ocular history were updated and adverse events were recorded.

Patient compliance to the treatment was reviewed during each visit by the unmasked examiner who was not involved in any of the safety or tolerability assessments.

Ophthalmological examination, including BCVA measurement, slit-lamp biomicroscopy with and without fluorescein, and IOP measurements, were performed. Subjects were asked to score ocular symptoms using the self-administering questionnaire described above. Ophthalmological examinations were done by a masked ophthalmologist who had no access to the randomization schedule.

At the day 14 visit, subjects were instructed to stop medication with the study eye drops. At the end of the day 21 visit, subjects were exited from the study.

\section{Efficacy phase}

The tolerability profile assessment phase consisted of a 2-week treatment period, followed by 1-week of follow-up. Visits were performed at days 0 (Baseline), 3, 7, 14, and the follow-up visit at day 21 .

\section{Study population}

Twenty-one adult patients affected by moderate to severe chronic blepharitis of either sex were included in the study (21 patients, 42 eyes). Inclusion criteria were more than 18 years of age, signing of the informed consent, diagnosis of moderate to severe blepharitis/blepharoconjunctivitis defined by a minimum score of at least one for one of the lid signs, 
one of the conjunctival signs, and one of the symptoms in at least one eye, and a minimum global score (total signs and symptoms score) of 5 in the same eye. ${ }^{16}$ The standardized validated photograph control scales used to evaluate blepharitis signs and symptoms were developed by Ora, Inc (Andover, MA, USA).

Exclusion criteria other than those mentioned for the tolerability profile were severe: dry eye syndrome, Schirmer test $<5 \mathrm{~mm}$, known endothelial corneal dystrophy, BCVA less than 0.1 decimal in either eye, and topical treatment with corticosteroids and/or antibiotics in the 2 weeks prior the inclusion visit or anticipated during the study.

\section{Efficacy endpoints/safety measures}

The primary efficacy outcome parameter of the study was a statistically significant reduction in the seven item global score, evaluated using Ora scales, in the efficacy study group between day 0 and day 14, and the difference between the global scores of the two groups at day 14. The seven-item global score was composed of the following signs and symptoms: lid margin redness (0-3), lid swelling (0-4), bulbar conjunctival redness (0-3), palpebral conjunctival redness $(0-3)$, ocular discharge $(0-3)$, itchy eyelids $(0-4)$, and gritty eyes (0-4).

Safety measures were measurement of BCVA, complete slit-lamp examination, dilated fundoscopy examination, IOP measurement (Goldmann tonometer), and pregnancy test where applicable.

Any adverse events were collected and reported.

\section{Inclusion visit (day 0)}

At the baseline visit, after signing of informed consent, one eye from each participant $(n=21)$ was included in the efficacy study group, the other eye $(n=21)$ in the efficacy control group. All eyes received treatment according to a computer-generated randomization schedule.

Medical and ocular history, concomitant medications and demographic data were recorded; women of childbearing potential underwent a urine pregnancy test. A complete ophthalmological evaluation was performed, including BCVA measurement, slitlamp biomicroscopy examination with and without fluorescein staining, Goldmann tonometry, dilated fundoscopy examination. Inclusion/exclusion criteria were reviewed.

The investigator rated the lid/conjunctival signs in each eye using the 0-3 (none to severe) standardized Ora scales. Subjects were asked to grade the frequency of the signs and symptoms of blepharitis using the Ora scale; the subject-reported signs and symptoms (lid swelling, itchy eyelids, and gritty eyes) were collected.

The study medication was dispensed and patients were instructed to clean the eyelid and eyelashes of both eyes with pre-moistened disposable wipes containing chlorhexidine and bisabolol every morning, and instill two drops of ophthalmic solution three times a day in the study eye, starting the morning after the visit, while in the control eye no medication had to be instilled.

\section{Visits after day 3, 7, I 4 and 2 I}

Medical and ocular history were updated, and adverse events were recorded.

Patient compliance to the treatment was reviewed in each visit by the unmasked examiner who was not involved in any of the efficacy or safety assessments.

Ophthalmological examination, including BCVA measurement, slit-lamp biomicroscopy with and without fluorescein, and IOP measurement was performed. Blepharitis signs and symptoms were recorded.

Ophthalmological examinations were performed by a masked ophthalmologist who had no access to the randomization schedule.

On the day 14 visit, subjects were instructed to stop medication and eyelid hygiene. At the end of the day 21 visit (follow-up), the patients were exited from the study.

\section{Statistical analysis}

For the efficacy phase we were planning a study of a continuous response variable from matched pairs of study subjects. Prior data indicated that the difference in the response of matched pairs was normally distributed with a standard deviation 0.4 . If the true difference in the mean response of matched pairs is 0.3 , we will need to study 21 pairs of subjects to be able to reject the null hypothesis that this response difference is zero with a probability (power) of 0.9 . The Type I error probability associated with this test of this null hypothesis is 0.05 . To calculate the sample size we used PS version 3.0.12 by Dupont WD and Plummer WD. ${ }^{17}$

The Wilcoxon signed-rank test for paired samples was used to compare the mean single sign/symptom score between the study and the control groups in either phase, and the mean global score and symptom score between the study group and the control group in the efficacy phase. The difference between the global score at day 0 and at the subsequent visits within each group was also analyzed. Statistical analysis was performed using Stata 10 (version 10, 2007; Stata Corp; College Station, TX, USA) $P<0.05$ was 
considered statistically significant. Results are expressed as mean \pm standard deviation. The statistical analysis was performed according to the intention-to-treat design.

\section{Results}

\section{Tolerability phase}

A total number of 20 volunteers were enrolled and randomized in the study, and all of them completed the study $(100 \%)$. There were 11 females and 9 males, with a mean age $45 \pm 19.9$ years (range: $18-90$ years), all of Caucasian race. Regarding primary tolerability parameters, there was no significant difference in the mean BCVA and IOP between groups at each visit, and no rise in IOP more than $4 \mathrm{mmHg}$ was reported (Table 1). No blurred vision was reported (data not shown) and no ocular signs of drug toxicity were reported.

Concerning subject-reported symptoms, there were statistically significant higher scores of tearing and ocular discomfort in the tolerability study group versus the tolerability control group during the treatment phase (day 0-14), and a significant higher mean score of itching in the tolerability study group at day 14 (Table 1).

Twelve patients reported ocular burning/stinging upon instillation, lasting a few seconds and of mild intensity.

No serious adverse events were reported during the course of the study in either group.

\section{Efficacy phase}

Twenty-one subjects were enrolled and randomized for the study, and 18 completed the study (85.7\%). There were 9 females and 12 males, mean age $55 \pm 21.1$ years (range: $20-83$ years), all of Caucasian race.

Three subjects withdrew from the study due to mild adverse events: two of them had eyelid erythema and swelling in the study eye, judged by the investigator as local hypersensitivity reaction, and they exited from the study at the day 14 visit; the other one at day 7 showed eyelid margin ulceration and progression of blepharitis in both eyes. These adverse events were considered mild events, and they resolved within a few days of treatment suspension and starting topical corticosteroid therapy.

No serious adverse events were reported during the course of the study in either group.

The baseline (day 0) global scores of blepharitis signs and symptoms, evaluated by standardized scales, were statistically lower in the efficacy control group in comparison to the efficacy study group $(P=0.005)$. From the day 3 visit to the day 14 visit, the differences between groups were not statistically

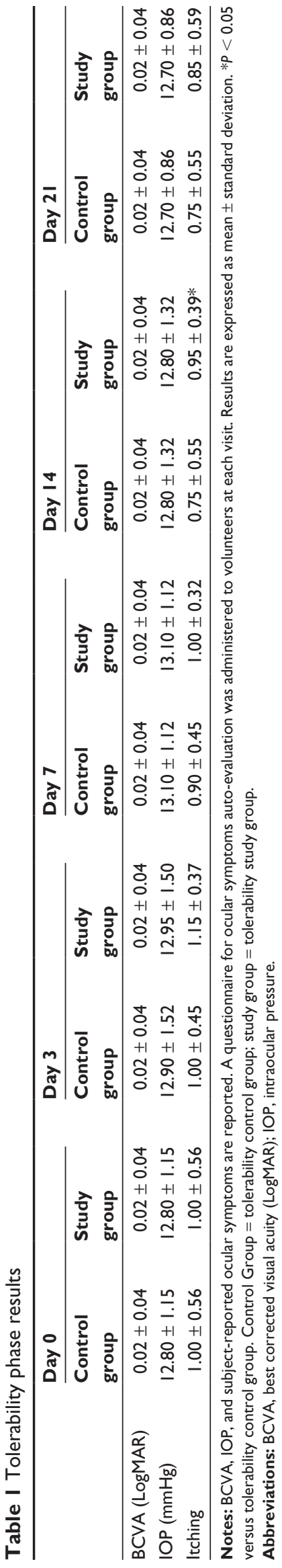


significant, even if a reduction in mean global score of the efficacy study group compared to the efficacy control group was evident. At the day 21 visit (follow-up visit), the mean score of the efficacy study group was significantly lower than the efficacy control group $(P<0.001)$. Results are reported in Table 2 and Figure 1.

The differences in global scores at days 3, 7, 14 and 21 versus baseline (day 0 ) were statistically significant in both groups $(P \leq 0.001)$.

\section{Discussion}

Blepharitis is one of the most common ocular surface pathologies and is recognized as one of the leading cause of patient referrals to ophthalmologists.

Nevertheless, at the moment there is no "institutional" consensus on the most effective therapy of this disease, probably due to its complex physiopathology, its various clinical presentations, and the frequent association with other ocular or systemic conditions. ${ }^{1}$ Currently, the management of blepharitis involves anti-inflammatory/antibiotic agents, eyelid hygiene, warm compresses, and artificial tears/lubricants. Topical corticosteroids are often used for the management of severe inflammation associated with blepharitis, and can offer patients rapid relief of symptoms. As their prolonged use can cause side-effects, such as IOP increase and cataract formation, they should only be used in short courses at the minimal dose. Given the frequent exacerbations and the severe chronic inflammation often occurring in blepharitis patients, sometimes not adequately managed with eyelid hygiene and artificial tears, the finding of new compounds that can exert anti-inflammatory activity without relevant side-effects is now considered more urgent.

Recently, the 18-beta-glycyrrhetic acid, the triterpene structure of the HMGB1-binding compound glycyrrhizin, has been extensively studied. ${ }^{13}$

Clinically, glycyrrhizin has been used for 20 years in Japan for the treatment of the viral hepatitis (hepatitis virus B and C), ${ }^{18,19}$ and studies have demonstrated that glycyrrhizin affords protection in a mouse model of hepatitis B by impairing the HMGB1-dependent recruitment of immune cells in the liver. ${ }^{20}$

Recent studies indicate an active role of HMGB1 release in inflammatory/degenerative pathologies of the eye: the protein is released in the aqueous humor or vitreous during experimental autoimmune processes, such as uveoretinitis in the rat, ${ }^{10}$ or bacterial infection such as endophthalmitis in humans $;{ }^{11}$ increased levels of extracellular HMGB1 have also been found in the vitreous of patients affected by proliferative diabetic- or vitreo-retinopathy. ${ }^{12}$ Recently, it was also found that the concentration of HMGB1 in subjects affected by bacterial or allergic seasonal conjunctivitis is 15 -fold higher than in healthy human subjects, and in patients with blepharitis it is 10 -fold higher. ${ }^{4}$

18b-glycyrrhetic acid was also reported to impair antibody recognition of $\mathrm{HMGB} 1$, suggesting direct binding, to prevent HMGB1-dependent COX2 expression and cluster formation in primary cultures human macrophages. ${ }^{4}$

The purpose of our study was to evaluate for the first time the tolerability profile in healthy volunteers and the efficacy in subjects affected by moderate/severe blepharitis of a novel medical device 5\% glycyrrhizin and copolymer PEG/PPG ophthalmic solution (DMG Italia SRL).

In the tolerability evaluation phase, no clinical signs of clinical toxicity or hypersensitivity, no rise in IOP, and no serious adverse event were reported. There was a statistically significant higher complaint of ocular discomfort in the tolerability study group compared to the control group and almost half of patients noticed a mild burning upon instillation, lasting only few seconds. These symptoms were of mild intensity and never forced the subjects to stop the treatment.

Concerning the evaluation of the efficacy phase, the protocol for both the efficacy study and efficacy control groups included eyelid hygiene with moistened disposable wipes, a fundamental treatment option for blepharitis.

The difference between the mean global symptom and sign scores between groups (evaluated using standardized photographic scales) was statistically significant at day 0 (Baseline) and day 21 (follow up). Although at day 0 the

Table 2 Mean global sign and symptom scores of both groups at each visit of the efficacy phase

\begin{tabular}{|c|c|c|c|c|c|}
\hline & Day 0 & Day 3 & Day 7 & Day I4 & Day 21 \\
\hline $\begin{array}{l}\text { Efficacy study group } \\
\text { (mean } \pm S D)\end{array}$ & $19.05 \pm 3.14$ & $15.76 \pm 3.55$ & $12.86 \pm 5.03$ & $9.24 \pm 5.49$ & $6.79 \pm 2.01$ \\
\hline $\begin{array}{l}\text { Efficacy control group } \\
(\text { mean } \pm S D)\end{array}$ & $16.38 \pm 3.85$ & $14.19 \pm 3.34$ & $13.14 \pm 4.35$ & $9.95 \pm 2.71$ & $9.95 \pm 3.63$ \\
\hline$P$ & $0.005 *$ & 0.092 & 0.587 & 0.074 & $0.001 *$ \\
\hline
\end{tabular}

Notes: Results are expressed as mean \pm SD. $* P<0.05$ versus efficacy control group.

Abbreviation: SD, standard deviation. 


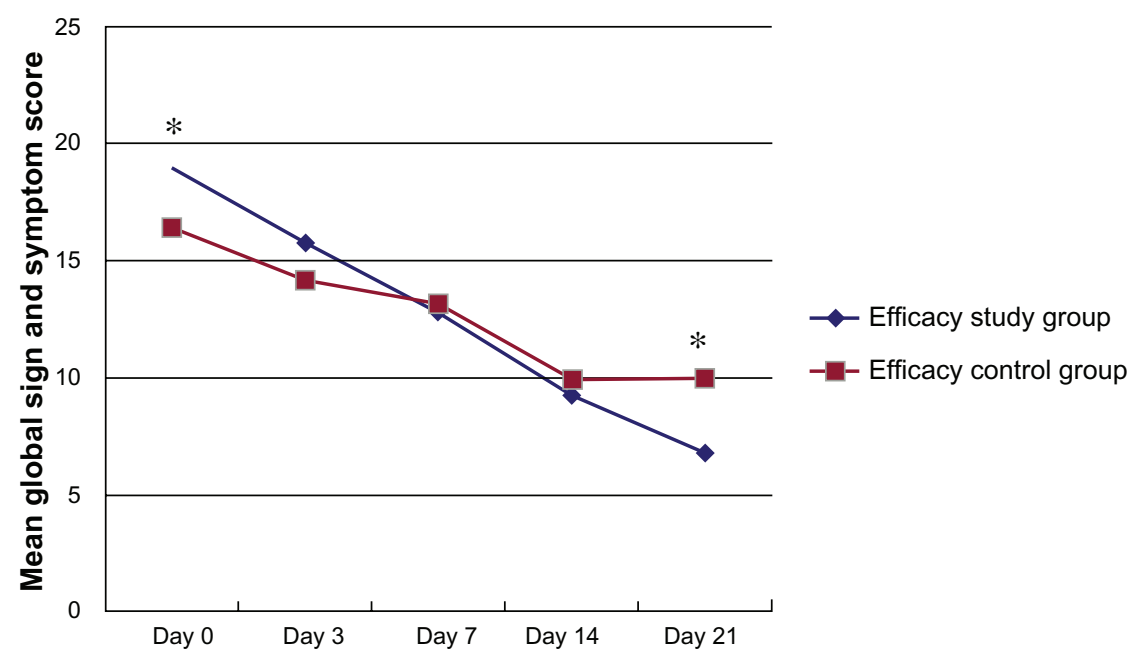

Figure I Mean global sign and symptom score in both groups at each visit of the efficacy phase.

Notes: Comparison of scores between Baseline (day 0$)$ and each subsequent visit were statistically significant in both groups $(P \leq 0.00 \mathrm{I})$. The differences between groups were statistically significant $(P<0.05)$ at day 0 and day 21 . Data are expressed as means. $* P<0.05$ versus efficacy control group.

eyes randomized to the efficacy study group showed a higher score (corresponding to a worse clinical picture at baseline) than the efficacy control group, the difference was no more statistically significant at day 14, and at day 21 (follow-up) there was an inversion, with a lower global score in the efficacy study group.

Even if the difference between the two groups was not statistically significant at the end of treatment (day 14), these results can be interpreted in our opinion as a higher efficacy of the glycyrrhizin ophthalmic solution compared to lid hygiene alone, lasting after treatment suspension.

The principal limitations of our study were the small number of subjects ( 20 healthy volunteers and 21 blepharitis patients), the open-label design, and the short duration of follow-up.

In conclusion, the 5\% glycyrrhizin ophthalmic solution was well tolerated in healthy volunteers and in patients with chronic moderate/severe blepharitis, and in association with eyelid hygiene showed good clinical anti-inflammatory activity, lasting after suspension.

Further clinical studies are necessary to evaluate the efficacy of the $5 \%$ glycyrrhizin and copolymer PEG/PPG ophthalmic solution in larger groups of patients for different periods of treatment and follow up. Given the high levels of HMGB1 also present in conjunctivitis, ${ }^{4}$ glycyrrhizin can be considered a promising therapeutic option for other inflammatory conditions of the ocular surface.

\section{Acknowledgments}

The authors thank Prof Riccardo Pistelli (Università Cattolica, Rome, Italy) for data management and statistics.
The authors also thank Matthew Chapin (Vice President Business Development Ora Inc, Andover, MA, US) for providing the Ora standardized photographic scales.

\section{Disclosure}

The clinical trial was sponsored by DMG Italia S.r.1. (Pomezia, Italy). The authors have no other conflicts of interest to declare.

\section{References}

1. American Academy of Ophthalmology Cornea/External Disease Panel Preferred Practice Pattern ${ }^{\circledR}$ Guidelines. Blepharitis PPP - Limited Revision - 2011 [webpage on the Internet]. San Francisco: American Academy of Ophthalmology; 2011. Available from: http://one.aao.org/ CE/PracticeGuidelines/PPP_Content.aspx?cid=500cd9ca-173c-4c31b6ea-a258e3549474. Accessed April 25, 2013.

2. Jackson WB. Blepharitis: current strategies for diagnosis and management. Can J Ophthalmol. 2008;43(2):170-179.

3. Foulks GN, Bron AJ. Meibomian gland dysfunction: a clinical scheme for description, diagnosis, classification, and grading. Ocul Surf. 2003;1(3):107-126.

4. Cavone L, Muzzi M, Mencucci R, et al. 18b-glycyrrhetic acid inhibits immune activation triggered by HMGB1, a pro-inflammatory protein found in the tear fluid during conjunctivitis and blepharitis. Ocul Immunol Inflamm. 2011;19(3):180-185.

5. Oppenheim JJ, Yang D. Alarmins: chemotactic activators of immune responses. Curr Opin Immunol. 2005;17(4):359-365.

6. Bianchi ME. DAMPs, PAMPs and alarmins: all we need to know about danger. J Leukoc Biol. 2007;81(1):1-5.

7. Dumitriu IE, Baruah P, Manfredi AA, Bianchi ME, Rovere-Querini P. HMGB1: guiding immunity from within. Trends Immunol. 2005;26(7): 381-387.

8. Lotze MT, Tracey KJ. High-mobility group box 1 protein (HMGB1): nuclear weapon in the immune arsenal. Nat Rev Immunol. 2005;5(4): 331-342.

9. Ulloa L, Messmer D. High-mobility group box 1 (HMGB1) protein friend and foe. Cytokine Growth Factor Rev. 2006;17(3):189-201.

10. Watanabe T, Keino H, Sato Y, Kudo A, Kawakami H, Okada AA. High mobility group box protein-1 in experimental autoimmune uveoretinitis. Invest Ophthalmol Vis Sci. 2009;50(5):2283-2290. 
11. Arimura N, Ki-I Y, Hashiguchi T, Sakamoto T, Maruyama I. Highmobility group box 1 protein in endophthalmitis. Graefes Arch Clin Exp Ophthalmol. 2008;246(7):1053-1058.

12. Pachydaki SI, Tari SR, Lee SE, et al. Upregulation of RAGE and its ligands in proliferative retinal disease. Exp Eye Res. 2006;82(5): 807-815.

13. Mollica L, De Marchis F, Spitaleri A, et al. Glycyrrhizin binds to highmobility group box 1 protein and inhibits its cytokine activities. Chem Biol. 2007;14(4):431-441.

14. Girard JP. A direct inhibitor of HMGB1 cytokine. Chem Biol. 2007; 14(4):345-347.

15. Akpek EK, Dart JK, Watson S, et al. A randomized trial of topical cyclosporin $0.05 \%$ in topical steroid-resistant atopic keratoconjunctivitis. Ophthalmology. 2004;111(3):476-482.

16. Torkildsen GL, Cockrum P, Meier E, Hammonds WM, Silverstein B, Silverstein S. Evaluation of clinical efficacy and safety of tobramycin/ dexamethasone ophthalmic suspension $0.3 \% / 0.05 \%$ compared to azithromycin ophthalmic solution $1 \%$ in the treatment of moderate to severe acute blepharitis/blepharoconjunctivitis. Curr Med Res Opin. 2011;27(1):171-178.
17. Dupont WD, Plummer WD. Power and sample size calculations. A review and computer program. Control Clin Trials. 1990;11(2): 116-128.

18. van Rossum TG, Vulto AG, de Man RA, Brouwer JT, Schalm SW. Review article: glycyrrhizin as a potential treatment for chronic hepatitis C. Aliment Pharmacol Ther. 1998;12(3):199-205.

19. van Rossum TG, Vulto AG, Hop WC, Schalm SW. Glycyrrhizin-induced reduction of ALT in European patients with chronic hepatitis C. Am J Gastroenterol. 2001;96(8):2432-2437.

20. Sitia G, Iannacone M, Muller S, Bianchi ME, Guidotti LG. Treatment with HMGB1 inhibitors diminishes CTL-induced liver disease in HBV transgenic mice. J Leukoc Biol. 2007;81:100-107.
Clinical Ophthalmology

\section{Publish your work in this journal}

Clinical Ophthalmology is an international, peer-reviewed journal covering all subspecialties within ophthalmology. Key topics include: Optometry; Visual science; Pharmacology and drug therapy in eye diseases; Basic Sciences; Primary and Secondary eye care; Patient Safety and Quality of Care Improvements. This journal is indexed on

\section{Dovepress}

PubMed Central and CAS, and is the official journal of The Society of Clinical Ophthalmology (SCO). The manuscript management system is completely online and includes a very quick and fair peer-review system, which is all easy to use. Visit http://www.dovepress.com/ testimonials.php to read real quotes from published authors. 